\title{
Comparative analysis of macrophage transcriptomes reveals a key mechanism of the immunomodulatory activity of Tricholoma matsutake polysaccharide
}

\author{
XIANG DING ${ }^{*}$, JIAN LI $^{*}$, YILING HOU and WANRU HOU \\ Key Laboratory of Southwest China Wildlife Resources Conservation, College of Life Sciences, \\ China West Normal University, Nanchong, Sichuan 637009, P.R. China
}

Received January 28, 2016; Accepted March 4, 2016

DOI: $10.3892 /$ or.2016.4814

\begin{abstract}
In the present study, we performed a proliferation assay, phagocytosis assay and cell cycle analysis of macrophages and sequenced the transcriptomes of control group macrophages and TMP-A group macrophages using Illumina sequencing technology to identify differentially expressed genes (DEGs) and determine the molecular mechanisms associated with differences in the immunomodulatory activity of TMP-A in macrophages. The results showed that TMP-A exhibits strong proliferation activity and phagocytosis activity in RAW264.7 cells in vitro and could also promote the proliferation of macrophage cells by abolishing cell-cycle arrest in the G0/G1 phase and promoting the cell cycle in the G2/M phase, which may induce cell division. A total of 12,616,096 and $11,798,839$ bp paired-end reads were obtained for the control group and TMP-A group, respectively, and they corresponded to a total size of $12.5 \mathrm{G}$ bp and $11.7 \mathrm{G} \mathrm{bp}$, respectively, after the low-quality reads and adapter sequences were removed. Approximately $79.8 \%$ of the total number of genes $(10,191)$ were expressed $(\mathrm{RPKM} \geq 1)$, and more than 1,372 genes were highly expressed (RPKM $>60$ ) in the TMP-A group. A total of 1,043 unigenes were identified as DEGs, and approximately 486 genes were upregulated, whereas 557 genes were downregulated, which might have contributed to the proliferation activity and phagocytosis activity of TMP-A in the RAW264.7 cells in vitro. A Gene Ontology (GO) enrichment analysis generated 13,042 assignments to cellular components, 13,094 assignments to biological processes, and 13,135 assignments
\end{abstract}

Correspondence to: Professor Yiling Hou, Key Laboratory of Southwest China Wildlife Resources Conservation, College of Life Sciences, China West Normal University, 1\# Shida Road, Nanchong, Sichuan 637009, P.R. China

E-mail: starthlh@126.com

\section{*Contributed equally}

Key words: polysaccharide, macrophage, mechanism, RNA-Seq, differentially expressed gene to molecular functions. A KEGG pathway enrichment analysis showed that the MAPK and $\mathrm{NF}-\kappa \mathrm{B}$ signaling pathways are significantly enriched for DEGs between the two cell groups. Based on the experimental data, we believe that the significant antitumor activities of TMP-A in vivo involve the MAPK and $\mathrm{NF}-\kappa \mathrm{B}$ signaling pathways because the two signaling pathways intersect.

\section{Introduction}

The efficacy of chemotherapeutic treatments for the majority of cancer types has improved in the last three decades, although the highly toxic effects of chemotherapeutic drugs still cause severe reductions in quality of life that present serious problems in clinical medicine (1). Therefore, developing effective low-toxicity anticancer drugs, including those based on natural products, is important. In recent years, polysaccharides from natural sources have received increasing attention as an efficient herbal medicine for the prevention and treatment of cancer because of their antitumor and immunomodulatory activities and low toxicity $(2,3)$. The antitumor properties are generally related to their ability to induce tumor cell apoptosis and activate macrophages (4).

Macrophages occupy a unique position in the immune system because they can initiate natural immune responses and then act as effector cells that help manage immune responses $(5,6)$, such as inflammation, angiogenesis and fighting an infection. Macrophages can eliminate the advanced stage of tumors because of their powerful functions, including phagocytosis and the release of numerous proinflammatory cytokines [interleukin (IL) and tumor necrosis factor (TNF)] and cytotoxic and inflammatory molecules [nitric oxide (NO) and reactive oxygen species (ROS)] that contribute to direct and/or indirect antitumor activities (7-9).

Recently, polysaccharides obtained from microorganisms, fungi and plants have become regarded as the most effective immune-regulating substances, and they have been shown to be clinically effective. Polysaccharides have anti-inflammatory, antihypoglycemic, antibacterial, and antitumor activities, and the basic mechanisms underlying the therapeutic effects of fungal polysaccharides, including their antitumor and immunostimulatory activities, likely occur through the modulation 
and stimulation of the complement system via macrophages (9). Since the discovery that Letinan, a polysaccharide from Lentinus edodes (Berk.) Sing, inhibited mouse sarcoma 180 and displayed low toxicity compared with chemical antitumor drugs (10), a number of polysaccharides with immunostimulatory and antitumor activities from species such as Coriolus versicolor, Agaricus blazei and Panax ginseng have been reported (11-13).

Tricholoma matsutake is a fungus belonging to the subgenus Tricholoma. As a traditionally edible fungus in Asian countries, particularly China, Japan and South Korea, Tricholoma matsutake has been used for the prevention and treatment of disease for several thousand years (14-16). Our group recently isolated a novel polysaccharide from Tricholoma matsutake named TMP-A, which has a backbone of 1,4- $\beta$-glucopyranose that branches at O- 6 , is composed of an $(1 \rightarrow 3)$ - $\alpha$-galactopyranose residue and terminates with an $\alpha$-xylopyranose residue (17). TMP-A also exhibits significant antitumor activities in vivo. However, the immunomodulatory activity and mechanism of TMP-A remain unclear. Here, we performed a proliferation assay, phagocytosis assay and cell cycle analysis of macrophages and sequenced the transcriptomes of macrophages of a control group and TMP-A group using Illumina sequencing technology. The goal of the present study was to identify differentially expressed genes (DEGs) in macrophages between the control group and TMP-A group to help determine the molecular mechanisms underlying the immunomodulatory activity of TMP-A in macrophages.

\section{Materials and methods}

Materials. The reagent 2-(2-methoxy-4-nitrophenyl-)3-(4nitrophenyl)-5-(2,4-disulfonic acid benzene)-2H-tetrazolium monosodium salt (CCK-8) was purchased from Dojindo Molecular Technologies, Inc. (Tokyo, Japan); lipopolysaccharide (LPS), D-Hanks solution, RPMI-1640 medium, fetal calf serum (FCS) and dimethyl sulfoxide (DMSO) were purchased from Gibco (Grand Island, NY, USA). Penicillin $\mathrm{G}$ and streptomycin were purchased from Sigma (Shanghai, China). All other chemicals and solvents were of analytical grade, and TMP-A was prepared in our laboratory as previously described (17).

Cell lines and reagents. The RAW264.7 cell line was cultured in RPMI-1640 medium containing $10 \%$ fetal bovine serum (FBS), 1\% penicillin (100 IU/ml) and streptomycin (100 mg/l) in a humidified atmosphere with $5 \% \mathrm{CO}_{2}$ at $37^{\circ} \mathrm{C}$ before use.

RAW264.7 cell proliferation assay. The cytotoxic effects of TMP-A on the RAW264.7 cells were determined by the CCK-8-based colorimetric method. Briefly, RAW264.7 cells suspended in RPMI-1640 medium at a density of $1 \times 10^{5}$ cells $/ \mathrm{ml}$ were pipetted into a 96 -well plate $(100 \mu \mathrm{l} /$ well $)$ and inoculated at $37^{\circ} \mathrm{C}$ in a humidified $5 \% \mathrm{CO}_{2}$ atmosphere. After incubation for $24 \mathrm{~h}, 100 \mu \mathrm{l}$ of the test sample at different concentrations was separately added into each well and incubated at $37^{\circ} \mathrm{C}$ in a humidified $5 \% \mathrm{CO}_{2}$ atmosphere for $48 \mathrm{~h}$. RPMI-1640 medium and $10 \mu \mathrm{g} / \mathrm{ml}$ LPS were used as the negative and positive controls, respectively. Subsequently, $20 \mu \mathrm{l}$ of CCK-8 reagent was added to each well, and the plate was further incubated for another 1-4 h. The absorbance of the colored solution at $490 \mathrm{~nm}$ was measured on a 96-well microplate reader (BioRad Laboratories, Tokyo, Japan). All of the experiments were performed in triplicate, and the inhibitory rate was calculated as follows: Cell proliferation activity $(\%)=[\mathrm{A} 2-\mathrm{A} 0] /[\mathrm{A} 1-\mathrm{A} 0] \mathrm{x}$ 100 where A 2 is the average optical density of TMP-A-treated cells, A0 is the average optical density of the control wells (culture medium without cells), and A1 is the average optical density of the negative control (culture medium containing cells). Each value is presented as the mean $\pm \mathrm{SD}(\mathrm{n}=4) ;{ }^{*} \mathrm{P}<0.05$ and ${ }^{* *} \mathrm{P}<0.01$ (vs. control).

RAW264.7 cell phagocytosis assay. RAW264.7 cells were inoculated in the presence of varying concentrations of TMP-A as described above. RPMI-1640 medium and LPS were used as the negative and positive controls, respectively. After $24 \mathrm{~h}$, the supernatants were removed, $100 \mu \mathrm{l}$ of $0.075 \%$ neutral red solution was added to each well, and the cells were cultured for an additional $1 \mathrm{~h}$. The plate was then washed three times with phosphate-buffered saline (PBS) and patted gently with tissues to allow the plates to drain. Finally, $100 \mu \mathrm{l}$ of cell lysis buffer $(0.1 \mathrm{~mol} / \mathrm{l}$ acetic acid and ethanol in a 1:1 ratio) was added to each well at $4^{\circ} \mathrm{C}$ for $2 \mathrm{~h}$. The absorbance at $540 \mathrm{~nm}$ was determined using a microplate ELISA reader. All of the analyses were conducted in triplicate. Each value is presented as the mean $\pm \operatorname{SD}(\mathrm{n}=4) ;{ }^{*} \mathrm{P}<0.05$ and ${ }^{* *} \mathrm{P}<0.01$ (vs. control).

RAW264.7 cell cycle analysis by flow cytometry. The effect of TMP-A on the cell cycle distribution was assessed by flow cytometry after staining the cells with propidium iodide (PI). RAW264.7 cells were seeded in 6 -well plates $\left(5 \times 10^{5}\right.$ cells/well) and allowed to grow for one day before being exposed to TMP-A $(1,5$ or $10 \mu \mathrm{g} / \mathrm{ml})$ for $72 \mathrm{~h}$. After incubation, the treated cells were harvested, washed twice with PBS and fixed in cold $70 \%$ ethanol for $4 \mathrm{~h}$ or overnight at $4^{\circ} \mathrm{C}$. After an additional wash in cold PBS, the cells were resuspended in $0.5 \mathrm{ml}$ of staining buffer containing $10 \mu \mathrm{l}$ of RNase and $25 \mu \mathrm{l}$ of PI, then incubated for $30 \mathrm{~min}$ in the dark at $37^{\circ} \mathrm{C}$. The DNA content of the cells was measured using a flow cytometer (Becton-Dickinson, Franklin Lakes, NJ, USA), and the population of cells in each phase was calculated using the ModFit LT software program. Each experiment was conducted three times.

RNA extraction, library preparation and sequencing. TRIzol reagent (Invitrogen, Burlington, ON, Canada) was used to extract the total RNA, and $1 \%$ agarose gels were used to investigate the RNA contamination and degradation. RNA purity was detected on a NanoPhotometer spectrophotometer (Implen, Inc., Westlake Village, CA, USA). After examining the RNA purity and concentration, the RNA 6000 Nano Assay kit with NanoDrop 2000 (Thermo Scientific NanoDrop $2000 \mathrm{c}$ ) was used to assess the RNA integrity. A total of $3 \mu \mathrm{g}$ of RNA per sample was used for the RNA sample preparations as input material (18). Following the manufacturer's recommendations, the transcriptome libraries were generated using the Illumina TruSeq ${ }^{\mathrm{TM}}$ RNA Sample Preparation kit (Illumina, San Diego, CA, USA). Clustering of the index-coded samples was completed using the TruSeq PE Cluster kit v3-cBot-HS (Illumina) on a cBot Cluster Generation System. The libraries 
were sequenced on an Illumina HiSeq 2000 platform after clustering, and $100 \mathrm{bp}$ paired-end reads were generated (18).

Transcriptome data analysis. In-house Perl scripts were used to process the raw data in FASTQ format to remove low quality reads, which contained poly-N stretches (partially un-sequenced regions) and adapter sequences. All of the downstream analyses are based on the high-quality clean sequences.

Differential expression and quantification analysis of the transcripts. Prior to performing the differential gene expression analysis, the read counts were adjusted using an edgeR program package for each sequenced library through one scaling normalized factor. The reads per kilobase per million reads (RPKM) method was used to quantify the transcript expression, and HTSeq v. 0.5.3 was used to count the number of reads mapped to each transcript. The RPKM value was calculated based on the mapped transcript fragments, sequencing depth and transcript length (18). The edgeR Bioconductor was used to complete the read counts with one scaling normalized factor before the analysis of differential gene expression, which was completed using the DEGSeq R package, release 1.12.0. A $\log 2$-fold change of \pm 1 and a P-value of 0.005 were set as the threshold of statistically significant differential expression. A large fold-change value (llog2-fold-changel $>5$ ) was also used to identify DEGs.

GO annotation and GO/KEGG enrichment analyses. The protein functions of all of the genes were annotated using BLASTX and InterProScan against the NCBI database. The resulting BLAST and InterPro annotations were then converted into Gene Ontology (GO) annotations. All of the GO terms were mapped to the GO slim categories. Fisher's exact test within Blast2GO [false discovery rate (FDR) <0.05] was used to determine the statistical significance of the functional GO slim enrichment. A hyper geometric test and the BenjaminiHochberg FDR correction were used to identify significantly enriched Kyoto Encyclopedia of Genes and Genomes (KEGG) pathways with KOBAS 2.0 (18).

\section{Results}

Proliferation of RAW264.7 cells following TMP-A treatment in vitro. The cytotoxicity or stimulation of TMP-A on RAW264.7 cells is shown in Fig. 1A and B. The cell proliferation activity was lowest when the macrophages were exposed to medium alone, whereas the incubation of these cells with increasing concentrations of TMP-A showed a dose-dependent increase in cell proliferation. The highest concentration of TMP-A significantly promoted RAW264.7 cell proliferation compared with the control group $(0.3125 \mu \mathrm{g} / \mathrm{ml}, \mathrm{P}<0.05$; $0.625-10 \mu \mathrm{g} / \mathrm{ml}, \mathrm{P}<0.01)$. Furthermore, the cell proliferation activity at a concentration of $10 \mu \mathrm{g} / \mathrm{ml}$ TMP-A was even greater than the activity elicited by $10 \mu \mathrm{g} / \mathrm{ml}$ LPS.

Phagocytosis activity of RAW264.7 cells following TMP-A treatment in vitro. The most striking feature of macrophage activation is the increase in pinocytic activity. The pinocytic activity of RAW264.7 cells following TMP-A treatment was
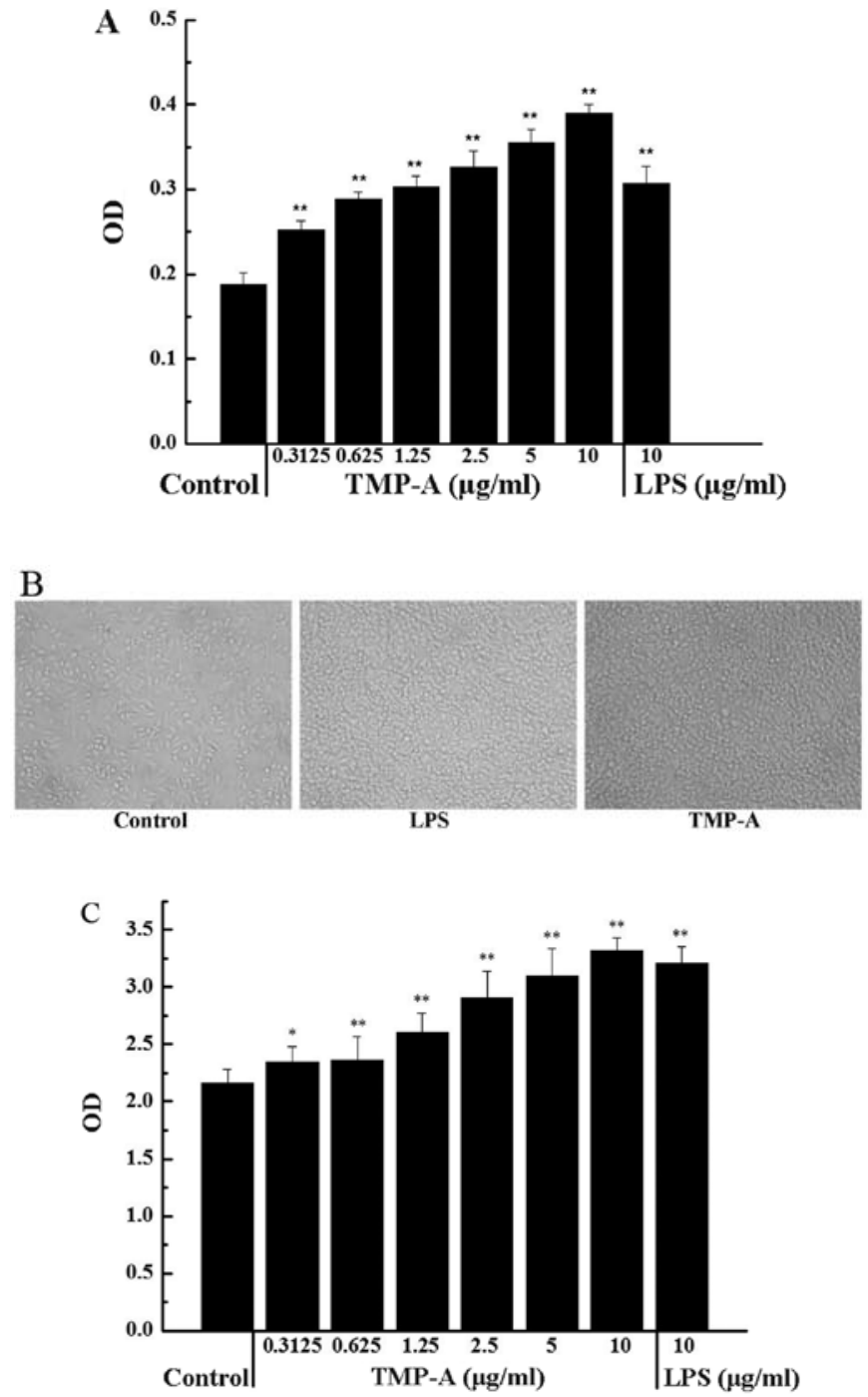

Figure 1. (A) Proliferation activity of RAW264.7 cells following TMP-A treatment in vitro. (B) Morphology of RAW264.7 macrophage cells in vitro. (C) Phagocytosis activity of RAW264.7 cells following TMP-A treatment in vitro. ${ }^{*} \mathrm{P}<0.05,{ }^{* *} \mathrm{P}<0.01$ compared with the control group.

examined by neutral red uptake activity $(0.075 \%)$. As shown in Fig. 1C, after 24-h incubation with varying concentrations of TMP-A, the phagocytosis activity of RAW264.7 was enhanced by TMP-A in the tested dose range in a dose-dependent manner compared with the negative control. Furthermore, the pinocytic activity at 5-10 $\mu \mathrm{g} / \mathrm{ml}$ TMP-A was comparable to or even greater than the activity elicited by $10 \mu \mathrm{g} / \mathrm{ml} \mathrm{LPS}$, a positive control.

Effects of TMP-A on the cell cycle distribution of RAW264.7 cells. To examine the effects of TMP-A on cell cycle progression, a cell cycle analysis was performed on RAW264.7 cells using flow cytometry. Fig. 2 shows the effects of TMP-A on the cell cycle phase (G0/G1,S and G2/M) distribution of RAW264.7 cells using flow cytometry with PI staining. The treatment of RAW264.7 cells with TMP-A at 2.5, 5 and $10 \mu \mathrm{g} / \mathrm{ml}$ for $72 \mathrm{~h}$ induced a significant and concentration-dependent increase in the $\mathrm{G} 2 / \mathrm{M}$ phase population from $17.1 \%$ of the control group to $15.3,16.6$ and $16.8 \%$, respectively $(\mathrm{P}<0.05$ or $\mathrm{P}<0.01)$, with a concomitant decrease in the percentage of cells in the G0/G1 

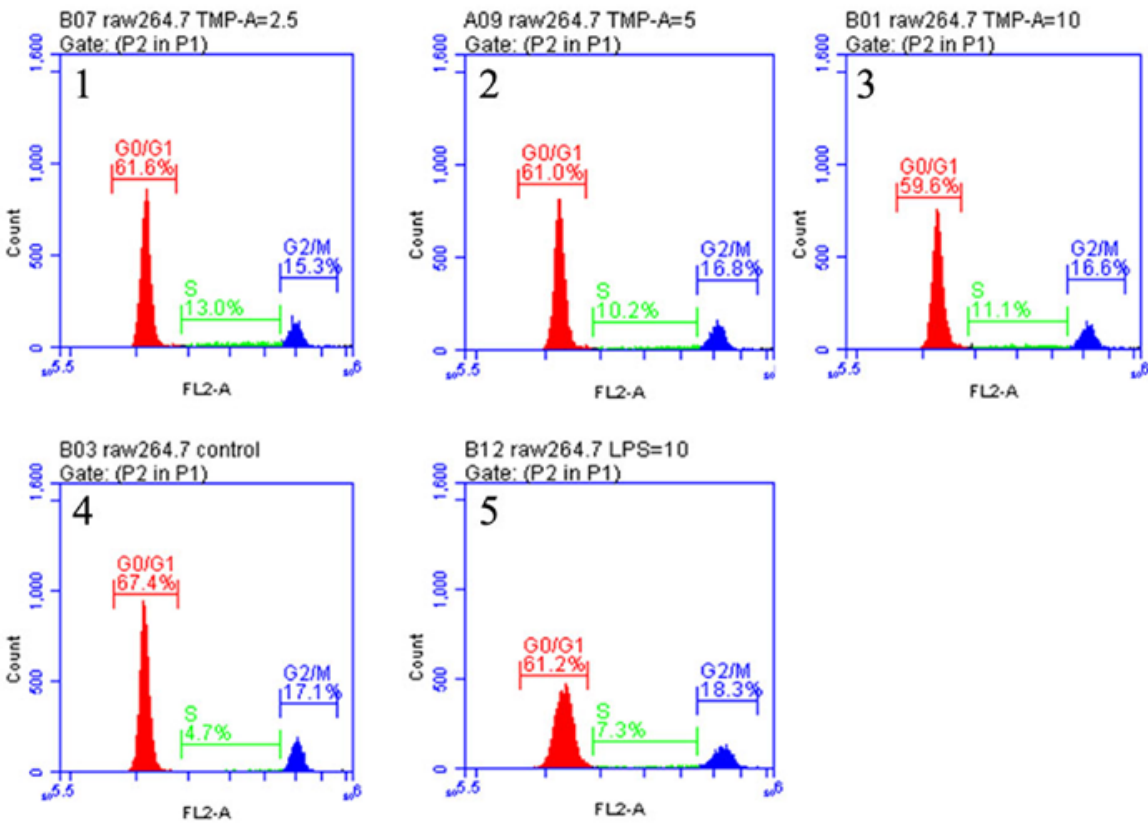

Figure 2. Effects of TMP-A on the cell cycle distribution of RAW264.7 cells. Each value is presented as the mean $\pm \mathrm{SD}(\mathrm{n}=4)$. $\mathrm{P}$-values are indicated as " $\mathrm{P}<0.05$ and ${ }^{* *} \mathrm{P}<0.01$ (compared with the control group).

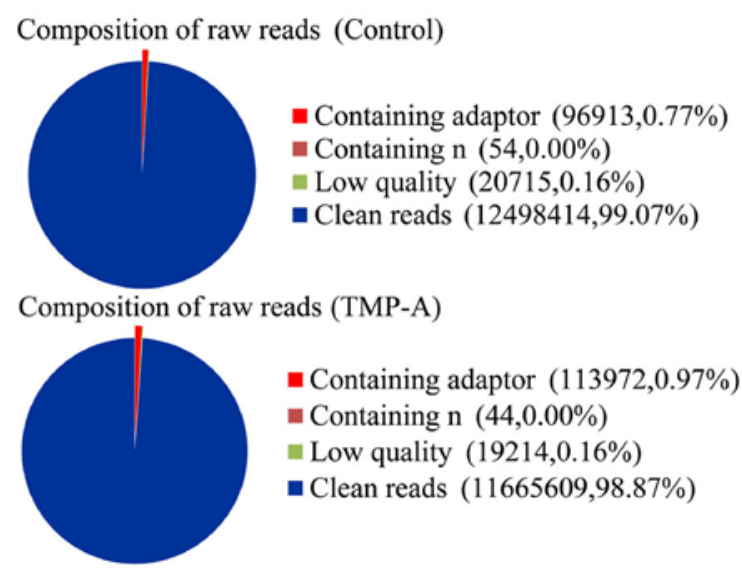

Figure 3. Quality assessment of the reads.

phase from $67.4 \%$ of the control group to $61.6,61.0$ and $59.6 \%$, respectively. At the tested concentrations, TMP-A also induced a significant change in the $\mathrm{S}$ phase population, from $4.7 \%$ of the control group to $13.0,10.2$ and $11.1 \%$, respectively. These results suggested that TMP-A could promote the proliferation of macrophage cells by abolishing cell cycle arrests in the G0/G1 and G2/M phases and promoting cell cycle progression in $\mathrm{S}$ phase, which might induce cell division.

Transcriptome sequencing and de novo assembly. To explore differences in the RAW264.7 cell transcriptomes between the control group and the TMP-A group, two cell groups were selected for analysis. Two cDNA libraries were constructed with the respective total RNA from the control group and TMP-A group. The prepared libraries were sequenced on an Illumina HiSeq 2000 platform. After quality control, a total of $12,616,096$ and $11,798,839$ bp paired-end reads were obtained for the control and TMP-A groups, respectively,
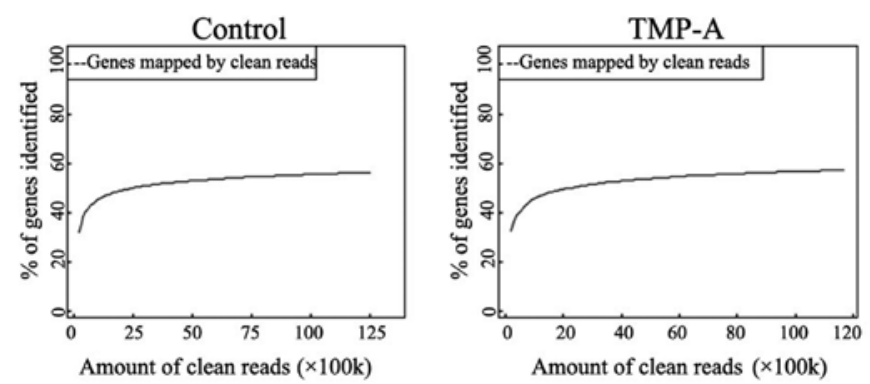

Figure 4. Sequencing saturation analysis.
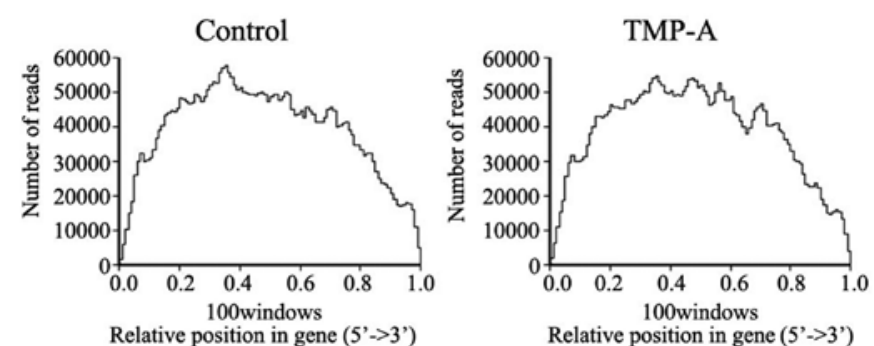

Figure 5. Randomness assessment.

which corresponded to a total size of $12.5 \mathrm{G} \mathrm{bp}$ and $11.7 \mathrm{G} \mathrm{bp}$, respectively, after the low-quality reads and adapter sequences were removed (Table I and Fig. 3). We mapped the clean reads to the RAW264.7 cell reference genome. The proportion of total reads in the two RAW264.7 cell transcriptome libraries that mapped to the genome ranged from 41.04 to $43.29 \%$. A sequencing saturation analysis showed that the number of genes detected by the library was saturated (Fig. 4). A 5'-3' sequence preference statistical analysis showed that the sequencing was mainly focused on the gene body region, and 
Table I. Summary of the mapping results (mapping to reference genome).

\begin{tabular}{lcccccccc}
\hline $\begin{array}{l}\text { Sample } \\
\text { ID }\end{array}$ & $\begin{array}{c}\text { Total } \\
\text { reads }\end{array}$ & $\begin{array}{c}\text { Total } \\
\text { base } \\
\text { pairs }\end{array}$ & $\begin{array}{c}\text { Total } \\
\text { mapped } \\
\text { reads }\end{array}$ & $\begin{array}{c}\text { Perfect } \\
\text { match }\end{array}$ & $\begin{array}{c}\leq 2 \mathrm{bp} \\
\text { mismatch }\end{array}$ & $\begin{array}{c}\text { Unique } \\
\text { match }\end{array}$ & $\begin{array}{c}\text { Multi-position } \\
\text { match }\end{array}$ & $\begin{array}{c}\text { Total } \\
\text { unmapped } \\
\text { reads }\end{array}$ \\
\hline Control & $12,498,414$ & $612,422,286$ & $5,129,004$ & 985,094 & $4,143,910$ & $3,166,326$ & $1,962,678$ & $7,369,410$ \\
& $(100.00 \%)$ & $(100.00 \%)$ & $(41.04 \%)$ & $(7.88 \%)$ & $(33.16 \%)$ & $(25.33 \%)$ & $(15.70 \%)$ & $(58.96 \%)$ \\
TMP-A & $11,665,609$ & $571,614,841$ & $5,050,408$ & 944,319 & $4,106,089$ & $3,045,421$ & $2,004,987$ & $6,615,201$ \\
& $(100.00 \%)$ & $(100.00 \%)$ & $(43.29 \%)$ & $(8.09 \%)$ & $(35.20 \%)$ & $(26.11 \%)$ & $(17.19 \%)$ & $(56.71 \%)$ \\
\hline
\end{tabular}

Table II. Quantification of gene expression in the control group (RPKM >10,000).

\begin{tabular}{|c|c|c|c|c|c|c|c|}
\hline $\begin{array}{l}\text { Gene } \\
\text { ID }\end{array}$ & $\begin{array}{c}\text { Uniq_reads } \\
\text { num }\end{array}$ & Length & Coverage & RPKM & Symbol & Description & $\begin{array}{l}\text { KEGG } \\
\text { orthology }\end{array}$ \\
\hline 171361 & 175276 & 1737 & $97.64 \%$ & 27073.88 & Eef1a1 & $\begin{array}{l}\text { Eukaryotic translation elongation } \\
\text { factor } 1 \propto 1\end{array}$ & K03231 \\
\hline 25319 & 49515 & 828 & $99.88 \%$ & 16044.80 & Fth1 & Ferritin, heavy polypeptide 1 & K00522 \\
\hline 81763 & 40233 & 1069 & $92.33 \%$ & 10097.94 & Rpl5 & Ribosomal protein L5 & K02932 \\
\hline 29304 & 39202 & 801 & $82.90 \%$ & 13131.18 & Rps6 & Ribosomal protein S6 & K02991 \\
\hline 116646 & 35323 & 794 & $95.59 \%$ & 11936.17 & Tpt1 & Tumor protein, translationally-controlled 1 & - \\
\hline 29282 & 29570 & 518 & $89.38 \%$ & 15316.15 & Rpl23 & Ribosomal protein L23 & K02894 \\
\hline 81776 & 24758 & 466 & $93.56 \%$ & 14254.68248 & Rps24 & Ribosomal protein S24 & K02974 \\
\hline 1406 & 19179 & 453 & $86.31 \%$ & 11359.41 & Rplp2 & Ribosomal protein, large P2 & K02943 \\
\hline
\end{tabular}

\section{Distribution of genes' coverage (Control)}

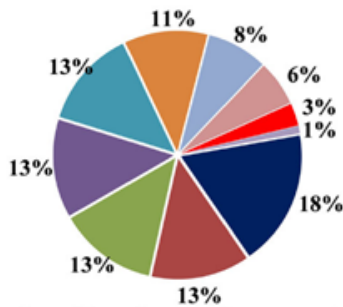

- $90 \%-100 \%(118)$ - $80 \%-90 \%(334)$ $=70 \%-80 \%(609)$ $=60 \%-70 \%(821)$ $=50 \%-60 \%(1068)$ $=40 \%-50 \%(1285)$ $=30 \%-40 \%(1340)$ $=20 \%-30 \%(1342)$ - $10 \%-20 \%(1328)$ $=0 \%-10 \%(1792)$

Distribution of genes' coverage (TMP-A)

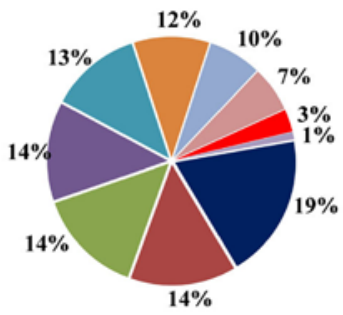

- $90 \%-100 \%(119)$ = $80 \%-90 \%(334)$ $=70 \%-80 \%(585)$ $=60 \%-70 \%(762)$ $=50 \%-60 \%(1063)$ $=40 \%-50 \%(1238)$

$=30 \%-40 \%(1332)$ $=20 \%-30 \%(1387)$ - $10 \%-20 \%(1387)$ $=0 \%-10 \%(1983)$

Figure 6. Distribution of gene coverage.

the bias at the two ends was limited (Fig. 5). The distribution of gene coverage is shown in Fig. 6 and provides a good basis for the follow-up analysis.

Transcriptome profiles of the two RAW264.7 cell groups. The abundance of all the genes was calculated and normalized using uniquely mapped reads by the RPKM method. The distribution of the expression levels of all the genes was similar for the two groups. Genes with RPKMs over 60 were considered to be expressed at a high level, whereas genes with RPKMs in the interval 0-1 were considered to be expressed at low levels or not at all. The results showed that in the control group, $\sim 81.8 \%$ of the total number of genes $(10,038)$ were expressed $(\mathrm{RPKM} \geq 1)$ and more than 1,333 genes were highly expressed (RPKM >60), whereas in the TMP-A group, $\sim 79.8 \%$ of the total number of genes $(10,191)$ were expressed (RPKM $\geq 1$ ) and $>1,372$ genes were highly expressed (RPKM $>60$ ).

The results also showed that eight genes (Eef1 $\alpha 1$, Fth1, Rpl23, Rps24, Rps6, Tpt1, Rp15 and Rplp2) were extremely highly expressed (RPKM >10,000) in the control group (Table II), whereas seven genes (Fth1, Eef1 $\alpha 1$, Rps24, Rpl23, Rps6, Rplp2 and Tpt1) were extremely highly expressed (RPKM $>10,000)$ in the TMP-A group (Table III). It is worth noting that the RPKM of the Fth1 gene was 16,044 in the control group and 78,552 in the TMP-A group. The Fth1 gene encodes the heavy subunit of ferritin, which is the major intracellular iron storage protein in eukaryotes and composed of 24 subunits of light and heavy ferritin chains. Changes in the ferritin subunit composition may affect iron absorption and release in different tissues. One of the major functions of ferritin is iron storage in a non-toxic and soluble state $(19,20)$. These results are consistent with the proliferation and phagocytosis activities of RAW264.7 cells following TMP-A treatment in vitro.

Differentially expressed genes between the control and TMP-A groups. The reads were adjusted using the edgeR program with one scaling normalized factor, and the DEGs 
Table III. Quantification of gene expression in the TMP-A group (RPKM > 10,000).

\begin{tabular}{lccccclcr}
\hline $\begin{array}{l}\text { Gene } \\
\text { ID }\end{array}$ & $\begin{array}{c}\text { Uniq_reads } \\
\text { _num }\end{array}$ & Length & Coverage & RPKM & Symbol & Description & $\begin{array}{c}\text { KEGG } \\
\text { orthology }\end{array}$ \\
\hline 25319 & 237268 & 828 & $99.88 \%$ & 78552.08078 & Fth1 & Ferritin, heavy polypeptide 1 & K00522 \\
171361 & 143447 & 1737 & $97.58 \%$ & 22638.13 & Eef1a1 & Eukaryotic translation elongation factor 1 $\alpha 1$ & K03231 \\
29304 & 34196 & 801 & $81.52 \%$ & 11702.85 & Rps6 & Ribosomal protein S6 & K02991 \\
116646 & 29917 & 794 & $95.59 \%$ & 10328.72 & Tpt1 & Tumor protein, translationally-controlled 1 & - \\
29282 & 27912 & 518 & $90.15 \%$ & 14771.01 & Rpl23 & Ribosomal protein L23 & K02894 \\
81776 & 26041 & 466 & $93.56 \%$ & 15318.65 & Rps24 & Ribosomal protein S24 & K02974 \\
140662 & 17295 & 453 & $86.31 \%$ & 10465.77 & Rplp2 & Ribosomal protein, large P2 & K02943 \\
\hline
\end{tabular}

Table IV. Differentially expressed genes: upregulated (llog2-fold-changel $>5$ ).

\begin{tabular}{lrclll}
\hline Gene ID & $\begin{array}{c}\text { Gene } \\
\text { length }\end{array}$ & $\begin{array}{c}\text { log2 ratio } \\
\text { (TMP-A/control) }\end{array}$ & Symbol & \multicolumn{1}{c}{ Description } & $\begin{array}{c}\text { KEGG } \\
\text { orthology }\end{array}$ \\
\hline 170496 & 876 & 13.15758 & Lcn2 & Lipocalin 2 & K01830; \\
& & & & & K03999 \\
287561 & 807 & 9.84101 & Cc17 & Chemokine (C-C motif) ligand 7 & K05509 \\
501162 & 645 & 9.109831 & Mreg & Melanoregulin & - \\
310969 & 2922 & 8.814769 & Ifi44 & Interferon-induced protein 44 & K13241 \\
24599 & 3793 & 8.784161 & Nos2 & Nitric oxide synthase 2, inducible & K01590 \\
24443 & 2367 & 8.743156 & Hdc & Histidine decarboxylase & K08283 \\
305475 & 3952 & 8.676823 & Osbp2 & Oxysterol binding protein 2 & K01104 \\
305064 & 4165 & 8.446367 & Ptpn14 & Protein tyrosine phosphatase, non-receptor type 14 & K14217 \\
56824 & 2174 & 8.411298 & Ifit1 & Interferon-induced protein with tetratricopeptide repeats 1 & K04459 \\
360580 & 1371 & 8.321534 & Dusp14 & Dual specificity phosphatase 14 & K00699 \\
113992 & 2351 & 7.935806 & Ugt1a6 & UDP glucuronosyltransferase 1 family, polypeptide A6 & K12965 \\
171091 & 1692 & 7.924924 & Zbp1 & Z-DNA binding protein 1 & K09877 \\
65054 & 1740 & 7.678115 & Aqp9 & Aquaporin 9 & K14217 \\
309526 & 2053 & 7.64592 & Ifit3 & Interferon-induced protein with tetratricopeptide repeats 3 & K05077 \\
171060 & 1915 & 7.539858 & Il13ra2 & Interleukin 13 receptor, $\alpha$ 2 & K05702 \\
292912 & 2355 & 7.241475 & Rasip1 & Ras interacting protein 1 & K15045 \\
65190 & 3628 & 7.165516 & Rsad2 & Radical S-adenosyl methionine domain containing 2 & K08341 \\
360468 & 3154 & 7.11958 & Emp2 & Epithelial membrane protein 2 & K12499 \\
81780 & 570 & 7.086246 & Ccl5 & Chemokine (C-C motif) ligand 5 & K09447 \\
293624 & 1985 & 7.010151 & Irf7 & Interferon regulatory factor 7 & K08017 \\
79253 & 3014 & 6.190835 & Avil & Advillin & K10104; \\
298698 & 1570 & 6.075358 & Angpt16 & Angiopoietin-like 6 & K05467 \\
64387 & 4114 & 5.180711 & Ccdc80 & Coiled-coil domain containing 80 & K04383 \\
24493 & 1992 & 5.045914 & Il1a & Interleukin 1 $\alpha$ &
\end{tabular}

between the two cell groups were identified using the DEGSeq $\mathrm{R}$ package. Values of FDR $\leq 0.001$ and $\log 2$ ratiol $\geq 1$ were set as the thresholds for significant differential expression. Our research group performed hierarchical clustering for all of the DEGs based on the $\log 10$ RPKMs of the two cells groups to observe the gene expression patterns (Fig. 7). A total of 1,043 unigenes were identified as DEGs, and 486 genes were upregulated, whereas 557 genes were downregulated (Fig. 7), which might have contributed to the proliferation and phagocy- tosis activities of RAW264.7 cells following TMP-A treatment in vitro. The numbers of DEGs in the control vs. TMP-A were 316 for transcripts detected with llog2-fold-changel $>2$ and 35 for transcripts detected with log2-fold-changel $>5$. Among the DEGs within the llog2-fold-changel $>5$ threshold, 24 genes were upregulated, including Ifi44, Ifit1, Ifit3, Il13r $\alpha 2$ and $\mathrm{Il1} \alpha$, among others, whereas 11 genes were downregulated, including RT1-Da, RT1-Db2 and Clqa, among others (Tables IV and Table V). 
Table V. Differentially expressed genes: downregulated (log2-fold-changel $>5$ ).

\begin{tabular}{lccllc}
\hline Gene ID & $\begin{array}{c}\text { Gene_ } \\
\text { length }\end{array}$ & $\begin{array}{c}\text { log2 ratio } \\
\text { (TMP-A/control) }\end{array}$ & Symbol & \multicolumn{1}{c}{ Description } & $\begin{array}{c}\text { KEGG } \\
\text { orthology }\end{array}$ \\
\hline 294269 & 1212 & -11.7903 & RT1-Da & RT1 class II, locus Da & K06752 \\
362634 & 1060 & -9.05405 & C1qc & Complement component 1, q subcomponent, C chain & K03988 \\
554353 & 1299 & -8.76072 & Gpr34 & G protein-coupled receptor 34 & K08383 \\
24981 & 1134 & -8.7343 & RT1-Db2 & RT1 class II, locus Db2 & K06752 \\
309621 & 1145 & -7.87593 & RT1-Ba & RT1 class II, locus Ba & K06752 \\
24499 & 4529 & -7.5208 & Il6r & Interleukin 6 receptor & K05055 \\
295283 & 2887 & -7.30369 & Ankrd34a & Ankyrin repeat domain 34A & - \\
171056 & 1318 & -6.26366 & Cx3cr1 & Chemokine (C-X3-C motif) receptor 1 & K04192 \\
291327 & 5091 & -6.09829 & Mrc1 & Mannose receptor, C type 1 & K06560 \\
361086 & 2886 & -5.58375 & Scel & Sciellin & K06084 \\
298566 & 1025 & -5.36135 & C1qa & Complement component 1, q subcomponent, A chain & K03986 \\
\hline
\end{tabular}

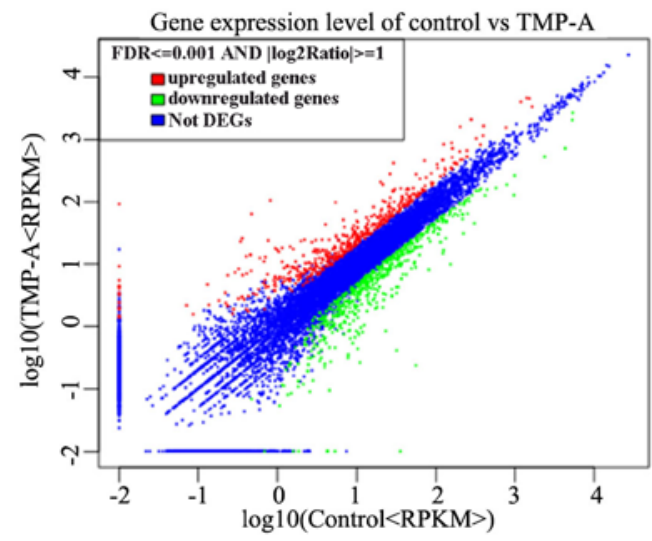

Figure 7. Scatter plot of differential expression.

IL-1 is involved in the regulation of immune response, inflammatory response and hematopoietic function. Interferons (IFNs) are a set of signals released by the host cells in response to pathogen release proteins, such as those from bacteria, parasites, viruses or tumor cells. IFNs belong to a category of proteins called cytokines, which protect and promote the immune system and help eliminate pathogens $(21,22)$.

IFNs also have a variety of other functions, including the activation of immune cells, such as macrophages and the regulation of the immune system. Both of these functions are important against antiviral infection. By interacting with specific receptors, IFNs activate signal transducer and activator of transcription (STAT) complexes. STATs are a family of transcription factors that regulate the expression of certain immune system genes. Type I IFNs further activate p38 mitogen-activated protein kinase to promote gene transcription (23). The antiproliferative and antiviral effects of type I IFNs are derived from p38 mitogen-activated protein kinase (MAPK) signaling. The phosphatidylinositol 3-kinase signaling pathway is also regulated by type I and II IFNs $(24,25)$. Based on experimental data and the results from our previous study, we believe that the significant antitumor activities of TMP-A in vivo may involve the MAPK signaling pathway of macrophages.
GO and KEGG enrichment analyses of the differentially expressed genes. GO analyses were used to confirm the functional classifications of the annotated unigenes and classify the transcripts with known proteins. A total of 39,271 genes were annotated with GO terms, which were converted to generic GO slim terms. The GO enrichment analysis was performed using Fisher's exact test in Blast2GO to analyze the gene functions of the DEGs. The analysis generated 13,042 assignments to cellular components, 13,094 assignments to biological processes, and 13,135 assignments to molecular functions. In the category of cellular components (Table III), 98.40 and $98.40 \%$ of the unigenes were located in cell parts (GO:0044464) and cells (GO:0005623), respectively. Most of the biological process categories were related to cellular processes (GO:0009987, 77.70\%) and metabolic processes (GO:0008152, 58.00\%). Under the molecular functions, the majority of the GO terms were grouped into binding (GO:0005488, 86.60\%) and catalytic activity (GO:0008152, $45.20 \%$ ) (Table VI). These results suggested that the immune mechanisms may present additional differences between the control group and TMP-A group.

The pathway analysis was conducted using the KEGG pathway database to further understand the biological function of the gene products. The KEGG pathway enrichment analysis was performed using KOBAS (KEGG Orthology Based Annotation System, v2.0). A KEGG analysis records the molecular interaction networks in cells with variants that are specific to particular organisms. We found that the MAPK signaling pathway (45 DEGs with pathway annotation: $5.10 \%$ ) (Fig. 8A) and the NF- $\kappa \mathrm{B}$ signaling pathway (20 DEGs with pathway annotation: $2.27 \%$ ) (Fig. $8 \mathrm{~B}$ ) are significantly enriched in the DEGs between the two cell groups (Table VII). This result also supported our previous hypothesis that the significant antitumor activities of TMP-A in vivo might involve the MAPK signaling pathway of macrophages and might also include the NF- $\kappa \mathrm{B}$ signaling pathway because there are intersections between the two signaling pathways.

It is worth noting that the cell cycle between the two cell groups (34 DEGs with pathway annotation: $3.85 \%$ ) is also significantly enriched for DEGs. These results indicated 
Table VI. Gene Ontology enrichment analysis of the DEGs.

\begin{tabular}{lccc}
\hline Gene Ontology term & \multicolumn{1}{c}{ Cluster frequency } & Genome frequency of use & Corrected P-value \\
\hline $\begin{array}{l}\text { Cell } \\
\text { GO:0044464 }\end{array}$ & 887 out of 901 genes, $98.4 \%$ & 12602 out of 13042 genes, $96.6 \%$ & 0.08999 \\
$\begin{array}{l}\text { Cell part } \\
\text { GO:0005623 }\end{array}$ & 887 out of 901 genes, $98.4 \%$ & 12602 out of 13042 genes, $96.6 \%$ & 0.08999 \\
$\begin{array}{l}\text { Cellular process } \\
\text { GO:0009987 }\end{array}$ & 682 out of 878 genes, $77.7 \%$ & 8844 out of 13094 genes, $67.5 \%$ & $8.64 \mathrm{e}-09$ \\
$\begin{array}{l}\text { Metabolic process } \\
\text { GO:0008152 }\end{array}$ & 509 out of 878 genes, $58.0 \%$ & 6505 out of 13094 genes, $49.7 \%$ & 0.00035 \\
$\begin{array}{l}\text { Binding } \\
\text { GO:0005488 }\end{array}$ & 753 out of 870 genes, $86.6 \%$ & 9931 out of 13135 genes, $75.6 \%$ & $4.17 \mathrm{e}-14$ \\
$\begin{array}{l}\text { Catalytic activity } \\
\text { GO:0008152 }\end{array}$ & 393 out of 870 genes, $45.2 \%$ & 4776 out of 13135 genes, $36.4 \%$ & $7.59 \mathrm{e}-06$ \\
\hline
\end{tabular}

Table VII. KEGG pathway enrichment analysis of the DEGs.

\begin{tabular}{lccccc}
\hline & $\begin{array}{c}\text { DEGs with } \\
\text { pathway } \\
\text { annotation (883) }\end{array}$ & $\begin{array}{c}\text { All genes } \\
\text { with pathway } \\
\text { annotation (13697) }\end{array}$ & P-value & Q-value & Pathway ID \\
\hline MAPK signaling pathway & $45(5.1 \%)$ & $357(2.61 \%)$ & $1.150435 \mathrm{e}-05$ & $1.794679 \mathrm{e}-04$ & ko04010 \\
NF-kB signaling pathway & $20(2.27 \%)$ & $144(1.05 \%)$ & 0.0009255778 & $6.016256 \mathrm{e}-03$ & ko04064 \\
Cell cycle & $34(3.85 \%)$ & $150(1.1 \%)$ & $7.470169 \mathrm{e}-11$ & $5.826732 \mathrm{e}-09$ & ko04110 \\
\hline
\end{tabular}

that TMP-A could promote the proliferation of macrophage cells by abolishing cell cycle arrest in the G0/G1 phase and promoting cell cycle progression in the G2/M phase, which might induce cell division.

\section{Discussion}

High-throughput and low-cost NGS technologies, such as RNA-Seq, have become popular and useful not only for de novo genome assembly and genome diversity studies but also to investigate gene expression profiles and discover pharmacological activity mechanisms. In our previous studies, the polysaccharide TMP-A exhibited significant antitumor activities in vivo. The inhibitory rate in mice treated with $80 \mathrm{mg} / \mathrm{kg}$ TMP-A reached $68.422 \%$, which might be comparable to the effects of mannatide. However, the immunomodulatory activity and mechanism of TMP-A remain unclear. Here, we performed a proliferation assay, phagocytosis assay and cell cycle analysis of macrophages, and the results showed that TMP-A exhibits strong proliferation and phagocytosis activities on RAW264.7 cells in vitro and could also promote the proliferation of macrophage cells by abolishing cell cycle arrest in the G0/G1 and G2/M phases and promoting cell cycle progression in $\mathrm{S}$ phase, which might induce cell division. To determine the mechanisms underlying the TMP-A effects on RAW264.7 cells and antitumor and immune activity, we sequenced the transcriptomes of macrophages of the control group and the TMP-A group using Illumina sequencing technology.

Our analysis identified 45 DEGs in the MAPK signaling pathway, 25 of which were upregulated in the TMP-A group (Fig. 8A), including EGF [K04357: 294559 (2.8)], c-Myc [K04377: 24577 (4.9)], IL-1 [K04383: 24493 (5.0); K04519: 24494 (3.5)] and TNF [K03156: 24835 (1.1)], whereas 20 were downregulated, including c-JUN [K03283: 24468 (-1.0)] and p38 [K04441: 81649 (-1.6)]. The MAPK signaling cascade is a common signal transduction module that connects different receptors/sensors tonuclear and cellular responses. The classical MAPK signaling cascade consists of three types of phosphorylated kinases: MAPK, MAPK kinase (MAPKK/MEK), and MAPK kinase kinase (MAPKKK/MEKK) (25). In the MAPK signaling pathway, EGF acts by binding to epidermal growth factor receptors (EGFRs) on the cell surface, which leads to cell proliferation, differentiation and survival. This process stimulates ligand dimerization and starts a signal transduction cascade reaction that results in a series of biochemical changes in cells as well as increased intracellular calcium levels, glycolysis and protein synthesis, with these changes eventually causing cell proliferation and DNA synthesis (26). This process would adequately explain the mechanism of the TMP-A proliferation activity on macrophages.

The IL-1 family is produced by macrophages, fibroblasts, monocytes, and these proteins play a significant role in the regulation of inflammatory and immune responses to infec- 


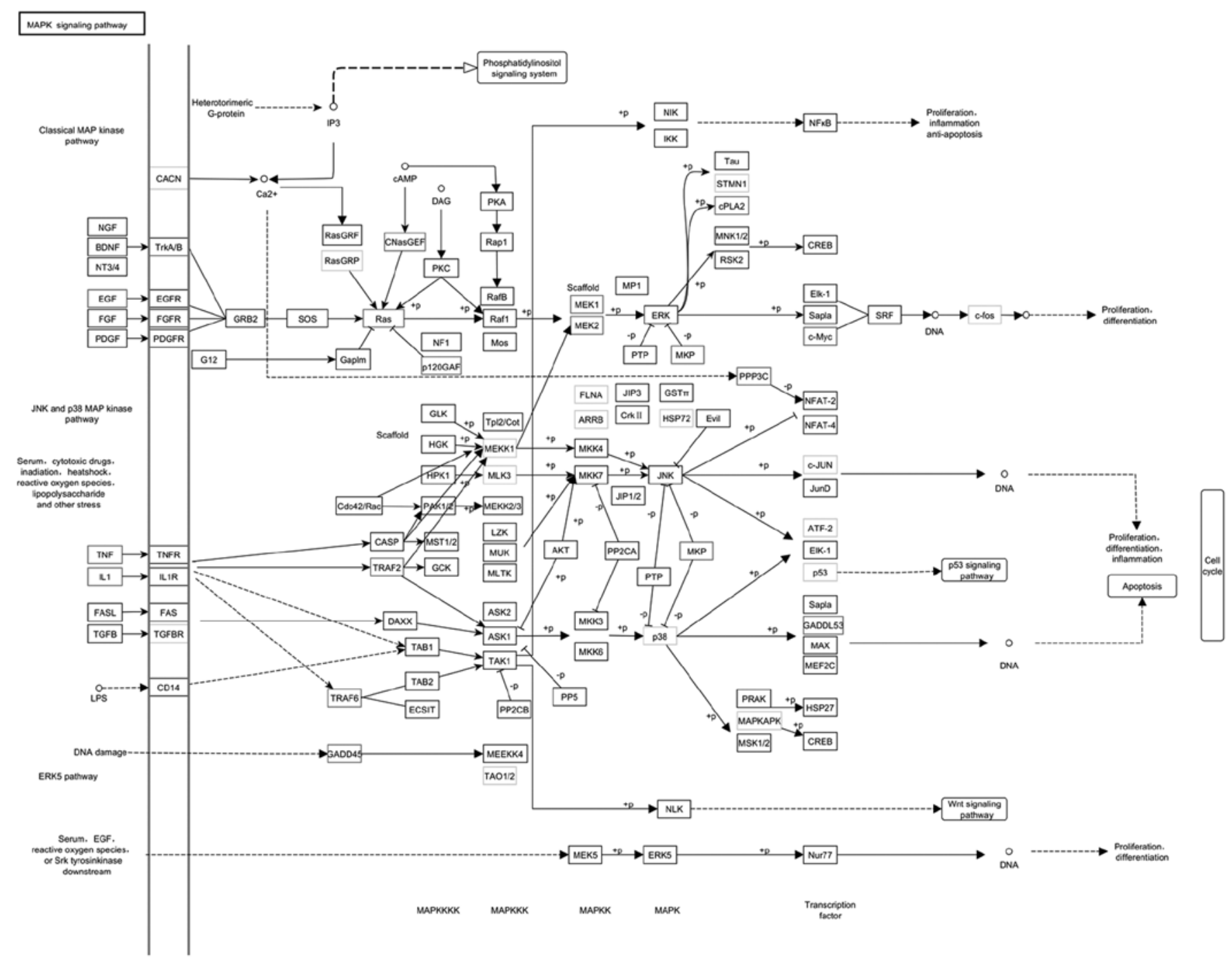

Figure 8. (A) MAPK signaling pathway.

tions. Thus, the upregulated DEGs in the MAPK signaling pathway in the TMP-A group might be associated with the high immunomodulatory activity of TMP-A on RAW264.7 cells in vitro.

Moreover, of the 20 DEGs in the NF- $\kappa \mathrm{B}$ signaling pathway (Fig. 8B), 17 were upregulated in the TMP-A group, including IL-1 $\beta$ [K04519: 24494 (3.5)], TNF $\alpha$ [K03156: 24835 (1.1)], TRAF6 [K03175: 114635 (1.0)] and COX2 [K11987: 29527 (2.2)], whereas only 3 were downregulated, including LTB [K03157: 361795 (-1.2)]. The NF- $\mathrm{B}$ family plays important roles in the immune system by regulating the expression of cytokines, inducible nitric oxide synthase (iNOS), cyclooxygenase 2 (COX-2) and growth factors. Under normal circumstances, the activation of $\mathrm{NF}-\kappa \mathrm{B}$ occurs because of the release of I $\mathrm{KB}$ molecules (27). In the classical activation pathway, signaling occurs by tumor necrosis factor receptor (TNFR), interleukin-1 receptor (IL-1R) and Toll-like receptors (TLRs). TNF $\alpha$ and IL-1 $\beta$ are the classic signaling molecules that can activate the I $\mathrm{B}$ kinase complex (28), which binds to other components and interacts with upstream signaling kinases. A number of stimuli can be produced by activating the IKK complex through different mechanisms, such as the phosphorylation of IKKs by upstream kinases or through the self-activation of IKK-dimers by mutual phosphorylation (29). IL-1 $\beta$ is a member of the IL- 1 cytokine family produced by macrophages, and it is an important mediator involved in a variety of cellular activities, including cell differentiation, cell proliferation and cell apoptosis (30).

TNF (which is also known as cachectin or TNF $\alpha$ ) is a cytokine with a wide variety of functions and is involved in the cytolysis of certain tumor cell lines, the induction of cachexia, and onset of fever by direct action or by stimulating IL-1 secretion. The upregulation of TNF genes in both the MAPK signaling pathway and the NF- $\mathrm{B}$ signaling pathway indicate that both pathways can be initiated $(31,32)$. First, in the activation of NF- $\mathrm{B}$, TRAF2 recruits the protein kinase IKK, which is activated by serine-threonine kinase (33). I $\kappa \mathrm{B} \alpha$ is an inhibitory protein that binds to $\mathrm{NF}-\kappa \mathrm{B}$ and prevents its translocation, and it is phosphorylated by IKK and degraded to release $\mathrm{NF}-\kappa \mathrm{B} . \mathrm{NF}-\kappa \mathrm{B}$ translocates into the nucleus and mediates the transcription of proteins involved in cell proliferation and survival (34). Second, during MAPK activation, TNF induces strong JNK activation and elicits p38-MAPK responses, which is important for ERK activation. TRAF2/Rac activates 


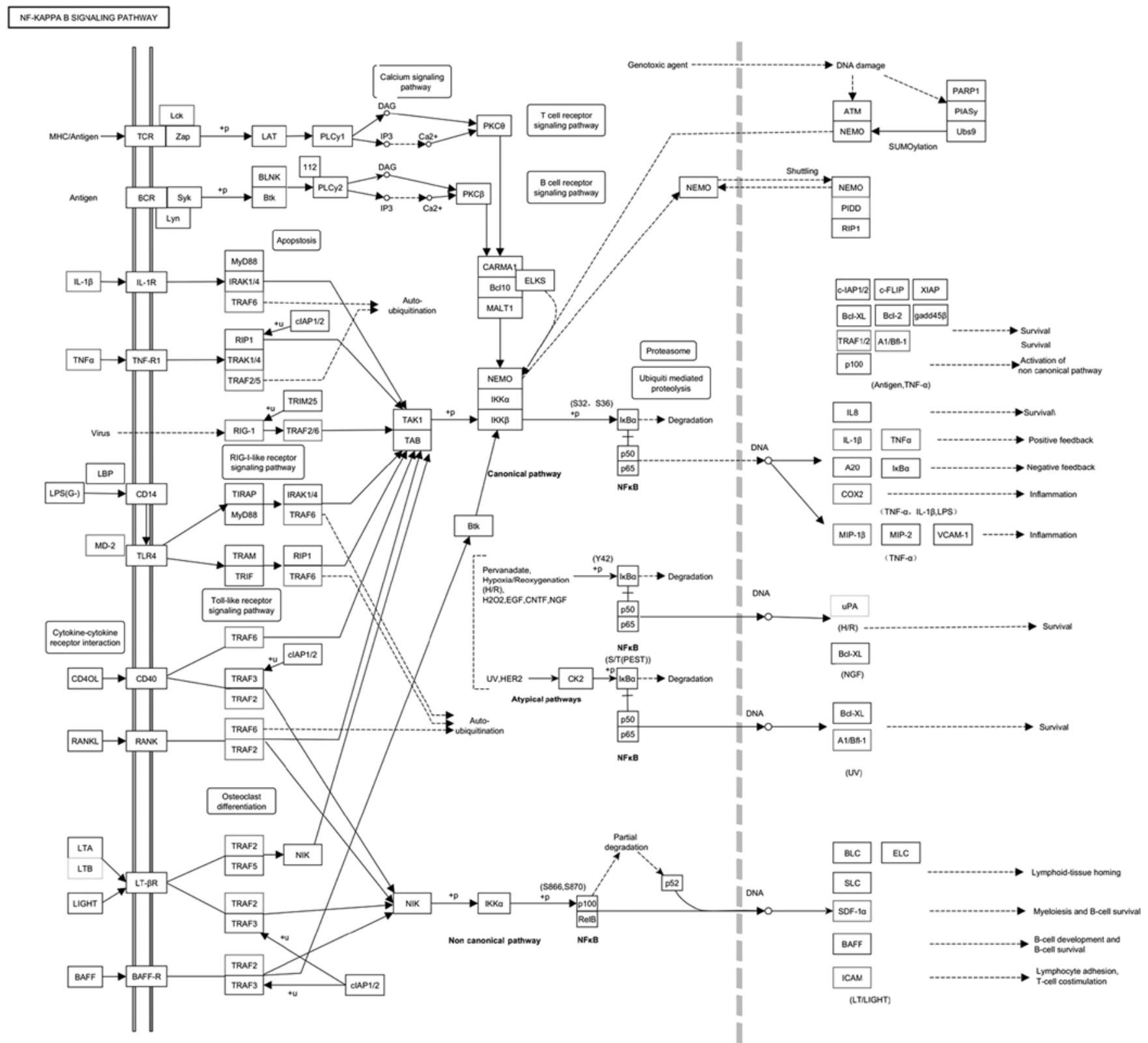

Figure 8. Continued. (B) NF-кB signaling pathway.

the upstream kinases of MEKK1 and MLK2/MLK3 induced by JNK (35), and then JNK translocates to the nucleus and activates transcription factors such as c-Jun and ATF2 $(36,37)$. The JNK pathway is also involved in cell differentiation, cell proliferation, and cell apoptosis. Thus, the upregulated DEGs in the TMP-A group might be associated with the strong effects of TMP-A on the proliferation activity, phagocytosis activity and cell cycle distribution of RAW264.7 cells in vitro, and these results can adequately explain the mechanism underlying the significant antitumor activities of TMP-A in the immune system.

In conclusion, we performed a proliferation assay, phagocytosis assay and cell cycle analysis of macrophages. Low cell proliferation activity and phagocytosis activity were observed when the macrophages were exposed to the medium alone, whereas a dose-dependent increase in cell proliferation activity and phagocytosis activity was observed after the cells were incubated with increasing concentrations of TMP-A. The cell proliferation activity induced by TMP-A was also time-dependent. The cell cycle analysis indicated that TMP-A could promote the proliferation of macrophage cells by abolishing cell cycle arrest in the G0/G1 and G2/M phases and promoting cell cycle proliferation in $\mathrm{S}$ phase, which might induce cell division. We then sequenced and characterized the transcriptomes of the macrophages of the control and TMP-A groups using Illumina sequencing technology, which enabled us to examine gene expression profiles and differential expression profiles and select functional genes related to the molecular mechanism of the immunomodulatory activity of TMP-A in macrophages. Based on the experimental data and the results from our previous study, we believe that the significant antitumor activities of TMP-A in vivo may involve the MAPK and NF- $\kappa \mathrm{B}$ signaling pathways because the two signaling pathways intersect. Our results provide a foundation 
for understanding the molecular mechanisms underlying the antitumor activity and immune activity of polysaccharides.

\section{Acknowledgements}

The present study was supported by the National Natural Science Foundation of China (31400016 and 31200012), the Application Foundation Project of Sichuan Province (2013JY0094), the Science and Technology Support Project of Sichuan Province (2014SZ0020 and 2014FZ0024), the Cultivate Major Projects of Sichuan Province (14CZ0016), the Open Foundation of Microbial Resources and Drug Development of Key Laboratory of Guizhou Province (GZMRD-2014-002), and the Doctor Startup Foundation Project of China West Normal University (11B019 and 11B020).

\section{References}

1. Rein DT and Kurbacher CM: The role of chemotherapy in invasive cancer of the cervix uteri: Current standards and future prospects. Anticancer Drugs 12: 787-795, 2001.

2. Song G and Du Q: Structure characterization and antitumor activity of an $\alpha \beta$-glucan polysaccharide from auricularia polytricha. Food Res Int 45: 381-387, 2012.

3. Schepetkin IA and Quinn MT: Botanical polysaccharides: Macrophage immunomodulation and therapeutic potential. Int Immunopharmacol 6: 317-333, 2006.

4. Zhao Z, Li J, Wu X, Dai H, Gao X, Liu M and Tu P: Structures and immunological activities of two pectic polysaccharides from the fruits of Ziziphus jujuba Mill. cv. jinsixiaozao Hort. Food Res Int 39: 917-923, 2006.

5. Lee KY and Jeon YJ: Macrophage activation by polysaccharide isolated from Astragalus membranaceus. Int Immunopharmacol 5: $1225-1233,2005$.

6. Song JY, Han SK, Son EH, Pyo SN, Yun YS and Yi SY: Induction of secretory and tumoricidal activities in peritoneal macrophages by ginsan. Int Immunopharmacol 2: 857-865, 2002.

7. Ma H, Liu G, Ding W, Wu Y, Cai L and Zhao Y: Diabetesinduced alteration of $\mathrm{F} 4 / 80^{+}$macrophages: A study in mice with streptozotocin-induced diabetes for a long term. J Mol Med Berl 86: 391-400, 2008.

8. Di Carlo E, Forni G, Lollini P, Colombo MP, Modesti A and Musiani P: The intriguing role of polymorphonuclear neutrophils in antitumor reactions. Blood 97: 339-345, 2001.

9. Cheng A, Wan F, Wang J, Jin Z and Xu X: Macrophage immunomodulatory activity of polysaccharides isolated from Glycyrrhiza uralensis Fish. Int Immunopharmacol 8: 43-50, 2008.

10. Chihara G, Maeda Y, Hamuro J, Sasaki T and Fukuoka F: Inhibition of mouse sarcoma 180 by polysaccharides from Lentinus edodes (Berk.) sing. Nature 222: 687-688, 1969.

11. Kobayashi H, Yoshida R, Kanada Y, Fukuda Y, Yagyu T, Inagaki K, Kondo T, Kurita N, Suzuki M, Kanayama N, et al: Suppressing effects of daily oral supplementation of beta-glucan extracted from Agaricus blazei Murill on spontaneous and peritoneal disseminated metastasis in mouse model. J Cancer Res Clin Oncol 131: 527-538, 2005.

12. Nakazato H, Koike A, Saji S, Ogawa N and Sakamoto J; Study Group of Immunochemotherapy with PSK for Gastric Cancer: Efficacy of immunochemotherapy as adjuvant treatment after curative resection of gastric cancer. Lancet 343: 1122-1126, 1994

13. Shin JY, Song JY, Yun YS, Yang HO, Rhee DK and Pyo S: Immunostimulating effects of acidic polysaccharides extract of Panax ginseng on macrophage function. Immunopharmacol Immunotoxicol 24: 469-482, 2002.

14. Gong MQ, Su LJ, Chen YW, Feng Z and Cao JX: A study on development of shiro and productive potentialities of Tricholoma matsutake. For Res 15: 374-379, 2002.
15. Guerin-Laguette A, Vaario L, Matsushita N, Shindo K, Suzuki K and Lapeyrie F: Growth stimulation of a Shiro-like, mycorrhiza forming, mycelium of Tricholoma matsutake on solid substrates by non-ionic surfactants or vegetable oil. Mycol Prog 2: 37-44, 2003.

16. Hur TC, Park H, Kang H and Joo SH: Dynamic changes of soil physicochemical properties in the fairy-rings of Tricholoma matsutake. J Korean For Soc 93: 26-34, 2004

17. Ding X, Feng S, Cao M, Li M, Tang J, Guo C, Zhang J, Sun Q, Yang Z and Zhao J: Structure characterization of polysaccharide isolated from the fruiting bodies of Tricholoma matsutake. Carbohydr Polym 81: 942-947, 2010.

18. Fang SM, Hu BL, Zhou QZ, Yu QY and Zhang Z: Comparative analysis of the silk gland transcriptomes between the domestic and wild silkworms. BMC Genomics 16: 60-72, 2015.

19. Zhang Y, Meng Q, Jiang T, Wang H, Xie L and Zhang R: A novel ferritin subunit involved in shell formation from the pearl oyster (Pinctada fucata). Comp Biochem Physiol B Biochem Mol Biol 135: 43-54, 2003.

20. Honarmand Ebrahimi K, Bill E, Hagedoorn PL and Hagen WR: The catalytic center of ferritin regulates iron storage via $\mathrm{Fe}(\mathrm{II})-$ Fe(III) displacement. Nat Chem Biol 8: 941-948, 2012.

21. Arko-Mensah J, Julián E, Singh M and Fernández C: TLR2 but not TLR4 signalling is critically involved in the inhibition of IFN-gamma-induced killing of mycobacteria by murine macrophages. Scand J Immunol 65: 148-157, 2007.

22. Zoon KC, Smith ME, Bridgen PJ, Anfinsen CB, Hunkapiller MW and Hood LE: Amino terminal sequence of the major component of human lymphoblastoid interferon. Science 207: 527-528, 1980.

23. Cooksley WG: The role of interferon therapy in hepatitis B. MedGenMed 6: 16, 2004.

24. Zarubin $\mathrm{T}$ and Han J: Activation and signaling of the p38 MAP kinase pathway. Cell Res 15: 11-18, 2005.

25. Orton RJ, Sturm OE, Vyshemirsky V, Calder M, Gilbert DR and Kolch W: Computational modelling of the receptor-tyrosinekinase-activated MAPK pathway. Biochem J 392: 249-261, 2005.

26. Herbst RS: Review of epidermal growth factor receptor biology. Int J Radiat Oncol Biol Phys 59 (Suppl): 21-26, 2004.

27. Gilmore TD: Introduction to NF-kappaB: Players, pathways, perspectives. Oncogene 25: 6680-6684, 2006.

28. Gilmore TD: The Rel/NF-kappaB signal transduction pathway: Introduction. Oncogene 18: 6842-6844, 1999.

29. Brasier AR: The NF-kappaB regulatory network. Cardiovasc Toxicol 6: 111-130, 2006.

30. Tian B and Brasier AR: Identification of a nuclear factor $\kappa$ B-dependent gene network. Recent Prog Horm Res 58: 95-130, 2003.

31. Locksley RM, Killeen N and Lenardo MJ: The TNF and TNF receptor superfamilies: Integrating mammalian biology. Cell 104: 487-501, 2001.

32. Gaur U and Aggarwal BB: Regulation of proliferation, survival and apoptosis by members of the TNF superfamily. Biochem Pharmacol 66: 1403-1408, 2003.

33. Wajant $\mathrm{H}$, Pfizenmaier $\mathrm{K}$ and Scheurich $\mathrm{P}$ : Tumor necrosis factor signaling. Cell Death Differ 10: 45-65, 2003.

34. Bouwmeester T, Bauch A, Ruffner H, Angrand PO, Bergamini G, Croughton K, Cruciat C, Eberhard D, Gagneur J, Ghidelli S, et al: A physical and functional map of the human TNF-alpha/ NF-kappa B signal transduction pathway. Nat Cell Biol 6: 97-105, 2004.

35. Chen G and Goeddel DV: TNF-R1 signaling: A beautiful pathway. Science 296: 1634-1635, 2002.

36. Baugh JA and Bucala R: Mechanisms for modulating TNF alpha in immune and inflammatory disease. Curr Opin Drug Discov Devel 4: 635-650, 2001

37. Lejeune FJ, Liénard D, Matter M and Rüegg C: Efficiency of recombinant human TNF in human cancer therapy. Cancer Immun 6: 6-16, 2006. 